\title{
Numerical Simulation of the Elastic Moduli of Cement Paste As a Three Dimensional Unit Cell
}

\author{
Ki-Bong Park \\ Division of Architecture College of Engineering, Kangwon national University, Chuncheon, S. Korea
}

\begin{abstract}
This paper describes a numerical method for estimating the elastic moduli of cement paste. The cement paste is modeled as a unit cell which consists of three components: the unhydrated cement grain, the gel, and the capillary pore. In the unit cell, the volume fractions of the constituents are quantified using a single kinetic function calculating the degree of hydration. The elastic moduli of cement paste are calculated from the total displacements of constituents when a uniform pressure is applied to the gel contact area. The cement paste is assumed to be a homogenous isotropic matrix. Numerical simulations were conducted through the finite element analysis of the three-dimensional periodic unit cell. The model predictions are compared with experimental results. The predicted trends are in good agreement with experimental observations. This approach and some of the results might also be relevant for other technical applications.
\end{abstract}

Keywords : Elastic Moduli, Hydration, Modeling, Finite Element Analysis

\section{INTRODUCTION}

Concrete, mortar and cement paste are usually made up of two or more phases that exhibit a non-linear mechanical behavior. Each material can be treated as a two-phase material in which aggregate particles are embedded in a homogenous matrix. Under this assumption, various investigators have proposed models and have derived expressions relating the elastic modulus of concrete to the elastic moduli and the volume concentration of the constituents. The elastic moduli of two-phase materials have been investigated by researchers in the field of crystallography. The first attempts to predict the moduli were carried out by Taylor (1983) and extended by Bishop and Hill (1951) for the yielding of a polycrystal composed of rigid-plastic single crystals. Their estimations were based on the nonlinear equivalents of the Voigt-Reuss bounds for linearelastic materials and depended only on the volume fraction of each phase. On basis of a theoretical expression, Kaplan (1959), Hill (1963), Hanshin and Shtrikman (1963), Counto (1964) proposed models for the prediction of the elastic moduli of concrete from the elastic moduli and the volume concentrations of the aggregate and matrix. These approaches, however, do not take into account any of the specific geometrical features of the phases and the stressfield interactions between neighboring particles. More accurate predictions, which included the effect of damage or phase changes for random reinforcement distributions, were obtained by Berveiller and Zaoui (1979). Zimmerman (1984) extended this approach to the more general case of elastic inclusions, arriving at a pair of differential equations which describe the change in elastic moduli as a function of inclusion concentration. Expressions for the elastic constants were obtained by solving the differential equations for this two-phase composite under the appro- priate boundary conditions. Tvergaad (1990) and Zhonghua et al. (1995) used a unit cell model, that considered "inclusions" having the same shape and dimensions, to investigate the dependence of tensile properties and flow behavior of particle-reinforced composites. Noda et al. (2005) investigated the effect of shape and arrangement of inclusions on the effective Young's modulus of heterogeneous materials through examining three types of unit cell models assuming 2D and 3D arrays of inclusions.

Another approach to this problem is a generalized differential effective medium theory (D-EMT) suggested by McLaughlin (1977). Garboczi and Berryman (2001) developed new D-EMT considering the number of particles of a certain type and its overlaps. The key idea was that each inclusion particle, surrounded by a shell of another phase, is mapped onto an effective particle of uniform elastic moduli. Roberts and Garboczi $(2001,2002)$ also have some sophisticated finite element simulations for computing the elastic modulus and other properties as well. The multi-phase model is appropriate to predict the elastic modulus, but for a cement-based material such as concrete or mortar, the material properties also depend upon the hydration process. To predict the elastic modulus more accurately, the multi-phase model must be combined with a hydration model.

In this paper, I focus on computing the elastic moduli of cement paste at the micrometer scale, by taking into account the microstructure at that scale. The three-phase composite model consisting of unhydrated cement, hydration products, and the pore space is used to model the cement-based material. After the hydration products contact the adjacent cement particles, the elastic modulus is calculated from the displacement change of cement paste with finite element method by applying uniform pressure on the contact area. The work in this paper provides a linkage 
between the physical-chemical changes occurring in the cement paste and the material's mechanical properties. The model can also be extended to look at the structural engineering of reinforced concrete

\section{UNIT CELL MODEL OF CEMENT PASTE}

A three-dimensional, cubic unit cell containing one identical sphere of cement particle uniformly distributed throughout the cell was generated using a new hydration model. It is necessary to know the volume growth rate of the microstructure using reasonable assumptions to explain the contact area between the hydration product, the pore, and the unreacted material. Our previous paper (Park. 2001; Park el al., 2006, 2008) described this volumetric growth rate as a function of the degree of hydration in a unit volume of cement based material. To simplify this aspect of the overall model, the volume of the cement particle was assumed to be occupied by a mix of three components, unreacted cement, a composite hydration product, and capillary pores. The cement particles were assumed to be spherical, uniformly sized, and uniformly distributed throughout the cement paste. As hydration progressed, the hydration products grew uniformly at the surface of a cement particle and so the overall shape remained spherical over time.

As shown in Figure 1, the model was formulated geometrically in terms of a unit cell. As the hydration products increased, the pore volume decreased. The basic elements of the model included the following:

The length of the cube that corresponds to the volume of cement pastes, $1 \mathrm{~cm}^{3}$, is:

$l=\sqrt[3]{4 \pi\left(\rho_{c} \chi+1\right) / 3 \quad r_{0}}$

where $\chi$ is water to cement ratio, $\rho_{c}$ is the density of cement $\left[\mathrm{g} / \mathrm{cm}^{3}\right]$, and the average radius of a cement particle, $r_{0}$ :

$r_{0}=\frac{3}{S \rho_{c}}$

where $S$ is the specific surface area of cement $\left[\mathrm{cm}^{2} / \mathrm{g}\right]$.

When the cement particle is not in contact with an adjacent particle, the degree of hydration, $\alpha$, is:

$\alpha=1-\left[\frac{r_{i}}{r_{0}}\right]^{3}$

where $r_{i}$ is the radius of the unhydrated cement particle $\left(=r_{0}-t\right)$ and $t$ is the layer depth of inner hydration products. The rate of volume growth of the hydration products, $\xi$, is:

$\zeta=\frac{R^{3}-r_{i}^{3}}{r_{0}^{3}-r_{i}^{3}}$

where $R$ is the radius of the cement particle including the outer hydration products. From equations (3) and (4), we find $R$ as a function of the degree of hydration and the rate of volume growth:

$$
R=[1+(\xi-1) \alpha]^{1 / 3} r_{0}
$$

The rate of volume growth was assumed have a constant value of 2.0. Therefore, $R$ can be calculated with the hydration model (Park el al., 2006) that describes the rate of hydration (Tomosawa, 1997). As shown in Figure $1, R$ changes gradually as the hydration reaction progresses. At any time, the volume of the cement paste including the outer hydration products, $V_{s}$, is:

$$
\begin{aligned}
& V_{s}=\frac{4}{3} \pi R^{3}, \quad R<\frac{l}{2} \\
& V_{s}=\frac{4 \pi R^{3}}{3}-6 \pi\left\{\frac{2}{3} R^{3}+\frac{l^{3}}{24}-\frac{R^{2} l}{2}\right\}, \quad \frac{l}{2}<R<\frac{\sqrt{2} l}{2} \\
& V_{s}=2 l^{2} \sqrt{R^{2}-\frac{l^{2}}{2}}+16 \int_{\sqrt{R^{2}-(l / 2)^{2}}}^{/ 2} S(x) d x, \quad \frac{\sqrt{2} l}{2}<R<\frac{\sqrt{3} l}{2}
\end{aligned}
$$

where $S(x)=S_{1}+S_{2}$

$S_{1}=\frac{l}{4} \sqrt{R^{2}-\left(\frac{l}{2}\right)^{2}-x^{2}}, \quad S_{2}=\frac{\left(R^{2}-x^{2}\right)}{2}\left[\frac{\pi}{4}-\arccos \left(\frac{l}{2 \sqrt{R^{2}-x^{2}}}\right)\right]$

At any time, $S c$ is the contact area of the hydration products including the outer hydration products. Sc changes gradually with $R$ during the hydration process according to the following equation:

$$
\begin{aligned}
& S_{c}=0,0<R<\frac{l}{2} \\
& S c=\pi\left\{R^{2}-\frac{l^{2}}{4}\right\}, \quad \frac{l}{2}<R<\frac{\sqrt{2} l}{2} \\
& S_{c}=8\left\{\frac{1}{2}\left[R^{2}-\left(\frac{l}{2}\right)^{2}\right]\left(\frac{\pi}{4}-\theta\right)+\frac{l}{4} \sqrt{R^{2}-\left(l^{2} / 2\right)}\right\}, \quad \frac{\sqrt{2} l}{2}<R<\frac{\sqrt{3} l}{2} \\
& \cos \theta=\frac{l}{2 \sqrt{R^{2}-(l / 2)^{2}}}
\end{aligned}
$$

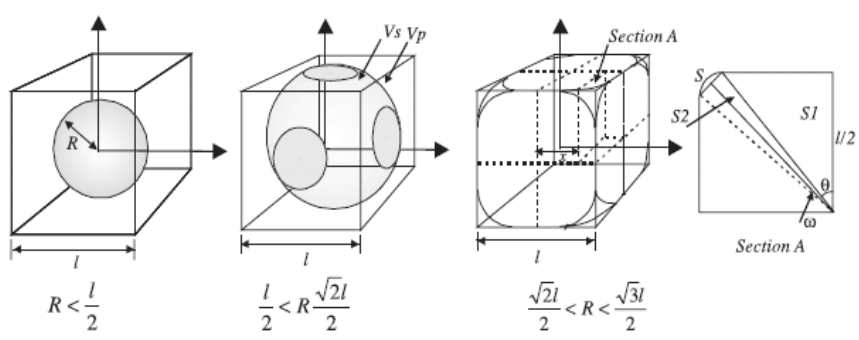

Figure 1. Microstructure formation as a function of the growth of hydration products 
The increase volume of the cement particle and the contact area between the outer radii of the cement particles is shown in Fig. 2 and Fig. 3 respectively. As a s chematic figure, the cube length is normalized to one.

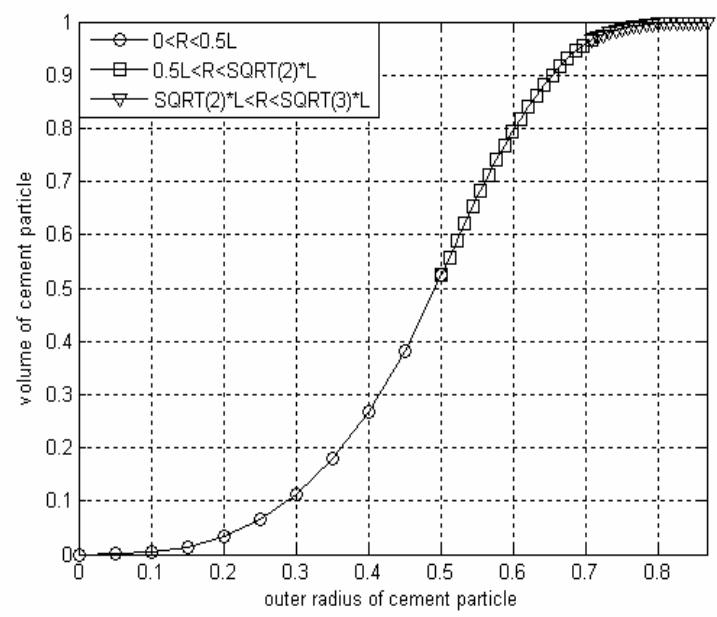

Figure 2. Volume of cement particle as a function of outer radius of cement particle

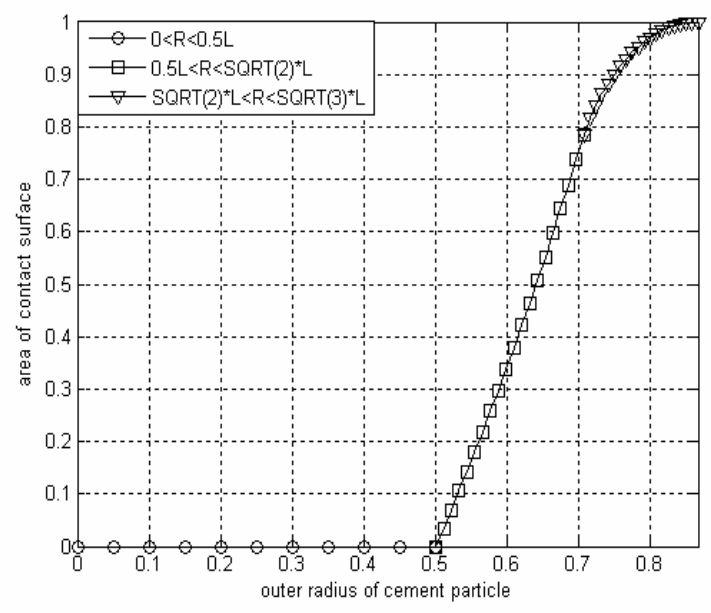

Figure 3. Area of contact surface as a function of outer radius of cement particle

\section{MODELING OF ELASTIC MODULUS OF CEMENT PASTE}

The property of a cement-based material is directly dependent on the degree of hydration. In this paper, the degree of hydration is the backbone for the description of all the evolution of all cement based materials properties. Based on an experimental regression of the degree of hydration of cement pastes, Hua et al. (1997) modeled the autogenous shrinkage of cement paste at the scale of the hydration grains. The cement particle was divided into some hydration process dependent continuous phase such as hydrates, residual anhydrous cement, capillary water and the transition zone, and the influence of capillary de- pression on autogenous shrinkage was studied. The research in this paper can be regarded as an extension of that hydration model. Based on the hydration model and the concept of multi-phase modeling, the elastic modulus of cement paste was predicted with a finite element method. The macroscopic bulk modulus of cement paste was modeled by assuming that each cement grain is spherical, and $t$ he gel was assumed to coat and cover all cement grain surfaces.

The unit model includes significant assumptions. Truly, the cement particles are distributed randomly and nonuniformly within the cement paste. But such a distribution would lead to a very time consuming and inefficient simulation. Therefore, in this paper, the space distribution of particles in cement paste is assumed homogeneous and the interaction between each particle is identical. Thus the hydration rate will be the same for all particles throughout the entire process and the volume growth rate of cement particle and pore structure formation will be easily evaluated.

As the cement hydration progresses, the connectivity among cement particles increase. At the initial setting-ti me, a connected solid system of cement particles is formed and the interparticle contact area begins to increase. Voigt et al. (2005) showed that the development of the compressive strength of cement paste is directly and linearly dependent on the specific effective contact area. Under the homogeneous assumption in this paper, the contact area between cement particles can be calculated using the hydration model. When the load is applied on the contact area, the contact area will be strained with the properties of composite materials and volume. As shown in Figure $4 \mathrm{~b}$, the elastic modulus is calculated by the following equation

$$
E=\frac{w l}{u(l * l)}=\frac{w}{u l}
$$

where

$w$ is the applied force on the contact area between two adjacent cement particles

$u$ is the displacement of the contact area

$l$ is the cube length and $l * l$ is the section area of the cement cube

In this study, the analytical simulation was based on a three-dimensional finite element method. The following assumptions were used to simulate the elastic modulus.

1. The cement paste consists of the unhydrated cement, hydration products, and pore space.

2. The unhydrated cement and the hydration product considered as different elastic stiffness.

3. The pore water, gel water, and vapor do not contribute to the mechanical strength of the composite. The elastic modulus of cement paste is only dependent upon the solid material in hydration process.

The numerical simulation details below: 


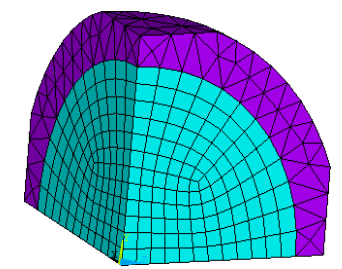

a. The grid of finite element model and material distribution

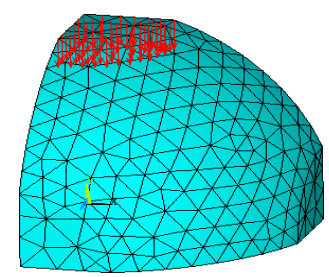

b. the applied uniform pressure on the contact area
Figure 4. The illustration of finite element model

\section{A: the material property and applied load}

According to the hypothesis of symmetry and periodicity of the cement particles, as shown in Figure $4 \mathrm{~b}$, a simplified one-eighth segment of the unit cell was used. The model is used to instead global cement particles.

This is equivalent to applying a uniform pressure on the contact area $x=l, y=l$ or $z=l$ ( $l$ is cube lengt h). In this paper, as shown in Figure $4 \mathrm{~b}$, the uniform pressure is applied on the contact area $y=l$.

As shown in Figure 4a, the cement particle is divid ed into two adjacent zones: unreacted cement (inner cyan color) and hydration products (outer purple color). As hydration progresses, the hydration product has a volume twice the original cement volume. The hydration products precipitate uniformly on the outer surface of the cement particle and the inner unreacted cement zone is successively replaced by hydration products.

When the unhydrated cement and hydrate are assumed to be homogenous and have an isotropic elasticity, at each step, a elastic modulus for the matrix is calculated with the tensor $A\left(E_{u h}, v_{u h}\right), A\left(E_{h}, v_{h}\right)$ (where $E_{u h}$ and $E_{h}$ is elastic modulus of unhydrated cement and hydrated cement respectively, $v_{u h}$ and $v_{h}$ are Poisson's ratio for the unhydrated and the hydrated cement respectively). The stiffness of the components in the structure varies considerably. According to Granju and Maso (1980), the cement grain has a modulus of elasticity of $60 \mathrm{GPa}$ as against 20 GPa for gel. Hua et al. (1997) reported that the elastic modulus of anhydrous cement is $40 \mathrm{GPa}$ and the elastic modulus for hydrates is $20 \mathrm{GPa}$. In this paper, we used 40 $\mathrm{GPa}$ and $25 \mathrm{GPa}$ respectively to fit the experimental data, The Poisson's ratios for the tensors $A\left(E_{u h}, v_{u h}\right), A\left(E_{h}, v_{h}\right)$ are equal and taken to be $v_{u h}=v_{h}=0.2$.

\section{B: boundary conditions.}

According to the hypothesis of periodicity and symmetry, the boundary conditions imposed on cement particle were:

On the contact area $x=l, y=l$ and $z=l$, the normal displacement of all nodes on each contact area were coupled respectively, and can be thought of as equivalent to a rigid plane imposed on the contact area. The boundary condition can be expressed as:

$$
\begin{aligned}
& u_{x} n_{x}=u_{x}(t) \\
& u_{y} n_{y}=u_{y}(t) \\
& u_{z} n_{z}=u_{Z}(t)
\end{aligned}
$$

On the faces $x=0, y=0$ and $z=0$, due to symmetr $\mathrm{y}$ of cement particle, the boundary conditions are:

$$
\begin{aligned}
& u_{x} n_{x}=0 \\
& u_{y} n_{y}=0 \\
& u_{z} n_{z}=0
\end{aligned}
$$

\section{Where}

$u_{x,} u_{y}, u_{z}:$ uniform displacement of each contact area re spectively, at a given time $t$

$n_{x}, n_{y}, n_{z}:$ normal vector of the contact area

\section{C: the element property}

In this paper, as shown in Figure 5, a 20-node isoparameter element was constructed to mesh the cement particle. This element can tolerate irregular shapes without much loss of accuracy, has compatible displacement shapes, and is well suited to model curved boundaries. Ea ch node of this element has three degrees of freedom: translations in the $\mathrm{x}, \mathrm{y}$, and $\mathrm{z}$ directions.

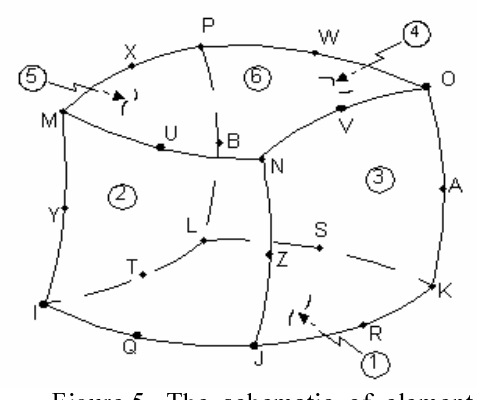

Figure 5. The schematic of element

\section{D: the solution procedure of elastic modulus}

The solution process for the elastic modulus was combined with the hydration model, and the calculation procedure followed:

$I^{0}$. according to the input parameter, such as the water to cement ratio, mineral composition of cement, the average radius of cement particles and the cement density, confirm the calculated coefficients for the hydration model.

$2^{0}$. according to the hydration equation, at each given time step, calculate the hydration degree of cement, confirm the relational calculation parameters for the elastic modulus, such as the radius of the unhydrated zone, and the outer radius of the cement particle. Establish the finite element model and calculate the elast ic modulus.

$3^{0}$. move on to the next time step, and carry out the iteration process 
Similar to Hua's paper (1997), this iteration process has some interesting advantages from an algorithmic viewpoint:

- The division number of finite element remains unchanged for whole calculation.

- The boundaries on which the boundary conditions are imposed remain unchanged.

- The elastic modulus is directly dependent on the hydration model, thus it is a programmed process and convenient to solve for the relational properties.

\section{E. calculation of elastic modulus}

In this part, the hydration process of an ordinary Portland cement (OPC) with a water to cement ratio 0.28 and 0.33 was studied. The mineral composition and hydration heat generation of this type cement are listed in Table 1 , and the hydration reaction coefficients are listed in Table 2.

Based on the hydration model and material properties, at times of 24 hours and 120 hours, the calcul ation parameters are listed in Table 3. Based on the calculation parameters for the elastic modulus in Table 3 and the finite element model, the elastic modulus was calculated and the calculated result is shown in Figure 6. It is obvious that calculated results agree well with the experimental curves. It proves the validity of this elastic modulus calculation model and the hydration model.

Table 1. Mineral composition and hydration heat generation of OPC.

\begin{tabular}{c|c|c|c|c}
\hline & $\mathrm{C} 3 \mathrm{~S}$ & $\mathrm{C} 2 \mathrm{~S}$ & $\mathrm{C} 3 \mathrm{~A}$ & $\mathrm{C} 4 \mathrm{AF}$ \\
\hline $\begin{array}{c}\text { Mineral Composi- } \\
\text { tion }\end{array}$ & 62 & 15 & 8 & 9 \\
\hline $\begin{array}{c}\text { Hydration Heat } \\
\text { generation }(\mathrm{J} / \mathrm{g})\end{array}$ & 500 & 260 & 866 & 420 \\
\hline
\end{tabular}

\begin{tabular}{c|c|c|c|c|c|c|c|c|c}
\multicolumn{8}{c}{ Table 2. Hydration reaction coefficients of OPC } \\
\hline $\begin{array}{c}\text { Cem. } \\
\text { W/C }\end{array}$ & $B_{20}$ & $C_{20}$ & $D_{20}$ & $k_{r 20}$ & $\beta_{1}$ & $\beta_{2}$ & $\beta_{3}$ & $E / R$ & $r_{0}$ \\
\hline $\mathrm{N} 28$ & $\begin{array}{c}3.5 \mathrm{e} \\
-10\end{array}$ & $\begin{array}{c}1.98 \\
\mathrm{e}-2\end{array}$ & $\begin{array}{c}3 \mathrm{e}- \\
11\end{array}$ & $\begin{array}{c}2.5 \mathrm{e} \\
-6\end{array}$ & 9 & 804 & 5942 & 6000 & 6.18 \\
\hline $\mathrm{N} 33$ & $\begin{array}{c}3.5 \\
\mathrm{e}-10\end{array}$ & $\begin{array}{c}1.71 \\
\mathrm{e}-2\end{array}$ & $\begin{array}{c}3 \mathrm{e}- \\
11\end{array}$ & $\begin{array}{c}2.5 \mathrm{e} \\
-6\end{array}$ & 9 & 804 & 5942 & 6000 & 6.18 \\
\hline
\end{tabular}

Table 3. The calculation parameter of elastic modulus

\begin{tabular}{c|c|c|c|c|c|c}
\multicolumn{6}{c}{ Table 3. The calculation parameter of elastic modulus } \\
\hline $\begin{array}{c}\text { Cem. } \\
\text { W/C } \\
\text { /Time }\end{array}$ & $l$ & $r_{i}$ & $\begin{array}{c}R \\
(\mathrm{um}) \\
(\mathrm{um})\end{array}$ & $\begin{array}{c}E_{u h} \\
(\mathrm{GPa})\end{array}$ & $\begin{array}{c}E_{h} \\
(\mathrm{GPa})\end{array}$ & $v$ \\
\hline $\mathrm{N} 28 / 24 \mathrm{~h}$ & 12.3 & 5.91 & 6.43 & 40 & 25 & 0.2 \\
\hline $\mathrm{N} 28 / 120 \mathrm{~h}$ & 12.3 & 5.6 & 6.67 & 40 & 25 & 0.2 \\
\hline $\mathrm{N} 33 / 24 \mathrm{~h}$ & 12.64 & 5.84 & 6.49 & 40 & 25 & 0.2 \\
\hline $\mathrm{N} 33 / 120 \mathrm{~h}$ & 12.64 & 5.46 & 6.77 & 40 & 25 & 0.2 \\
\hline
\end{tabular}

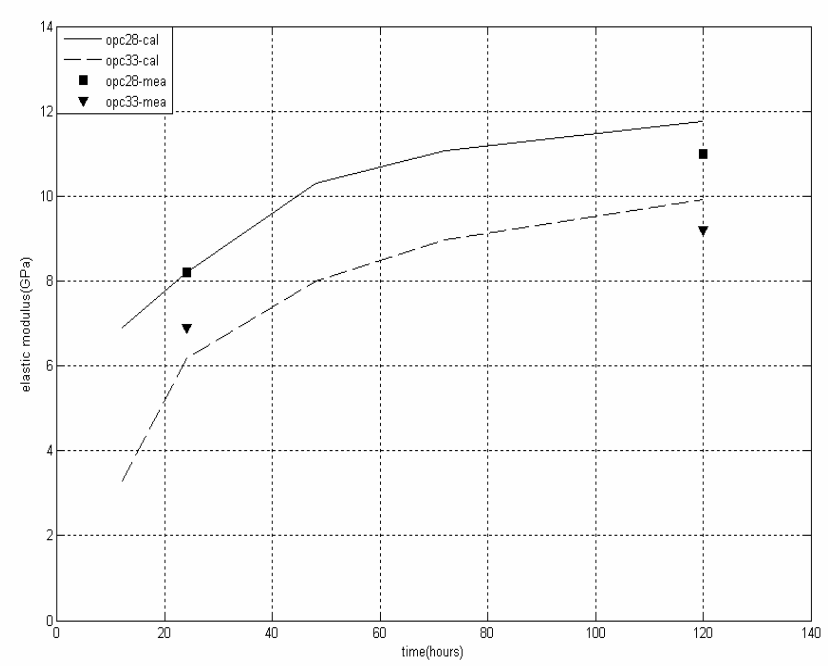

Figure 6. Calculated curves and experiment curves

\section{CONCLUSION}

Based on the kinetic hydration model and the concept of multi-phase modeling, a finite element model to predict the evolution of elastic modulus of cement paste was built and proved valid compared with experimental results.

The development of elastic modulus is directly dependent on the hydration process and the trend of elastic modulus is consistent with the heat evolution rate after the dormant period. Furthermore, based on the evolution of cement paste elastic modulus, the development of concrete elastic modulus can be predicted and the early age deformation of actual reinforced concrete structure under load effect can be estimated.

\section{ACKNOWLEDGEMENTS}

This research was supported by Basic Science Research Program through the National Research Foundation of Korea funded by the Ministry of Education, Science and Technology(331-2008-D00661)

\section{REFERENCES}

Berveiller, M. \& Zaoui, A. (1979) "An extension of the self-consistent scheme to plastically-flowing polycrytals" J. Mech. Phys. Solids 26, 325-344.

Bishop, J. F. W. \& Hill, R. (1951) “A theoretical derivation of plastic properties of a polycrystalline face-center metal" Philos. Mag. 42, 1298-1307.

Boumiz, A., Vernet, C. \& Tenoudji, F. C. (1996) "Mechanical properties of cement pastes and mortar at early ages - Evolution with time and degree of hydration" Adv. Cem. Basde Mater. 3, 94-106. 
Counto, U. J. (1964) "The effect of the elastic modulus of the aggregate on the elastic modulus, creep and creep recovery of concrete" Mag. Concr. Res. 16 (48), 129138.

Garboczi, E. J. \& Berryman, J. G. (2001) "Elastic moduli of a material containing composite inclusions: effective medium theory and finite element computations" Mech. Mater. 33, 455-470.

Granju, J. L. \& Maso, J. C. (1980) "Module de deformation longitudinal des pastes de ciment Portland conserves dans l'eau" Cem. Concr. Res. 10, 731-738.

Hashin, Z. \& Shtrikman, S. (1963) “A variational approach to the theory of the elastic behavior of multiphase materials" J. Mech. Phys. Solids 11 (2), 127-140.

Hill, R. (1963) "Elastic properties of reinforced solids: some theoretical principles" J. Mech. Phys. Solids 11 (5), 357-372.

Hua, C., Ehrlacher, A. \& Acker, P. (1997) “Analyses and models of the autogenous shrinkage of hardening cement paste II. Modelling at scale of hydration grains" Cem. Concr. Res. 27 (2), 245-258.

Kaplan, M. F. (1959) "Ultrasonic pulse velocity, dynamics modulus of elasticity, Poisson's ratio, and the strength of concrete made with thirteen different coarse aggregate" RILEM Bulletin. 1, pp. 58-73.

McLaughlin, R. (1977) "A study of the differential scheme for composite materials" Int. J. Eng. Sci. 15, 237-244.

Noda, N., Nisitani, H., Takase, Y. \& Shukuwa, Y. (2005) "Two-dimensional and axisymemetric unit cell models in the analysis of composite materials" Comp. Struc. 69, 429-435.

Park, K. B. (2001) "Prediction of cracking in high-strength concrete using a hydration model" $\mathrm{PhD}$ thesis, The University of Tokyo.

Park, K. B., Noguchi, T. \& Plawsky, J. (2005) "Modeling of hydration reactions using neural networks to predict the average properties of cement paste" Cem. Concr. Res. 35, 1676-1684.

Park, K. B., Jee, N. Y., Yoon, I. S. \& Lee, H. S. (2008) "Prediction of temperature distribution in high-strength concrete using hydration model" Mar.-Arp., 180-186.

Roberts, A. P. \& Garboczi, E. J. (2001) "Elastic moduli of model random three-dimensional closed-sell cellular solids" Acta. Mater. 49, 189-197.

Roberts, A. P. \& Garboczi, E. J. (2002) "Elastic moduli of model random three-dimensional closed-sell cellular solids" Mech. Phys. Solids 50, 33-35.

Taylor, G. I. (1983) "Plastic stains in metals" J. Inst. Metals 62, 307-624.

Tomosawa, F (1997) "Development of a kinetic model for hydration of cement" Proceedings of the $10^{\text {th }}$ ICCC, V, II, 8 .

Tvergaad, V. (1990) "Analysis of tensile properties for a whisker-reinforced metal-matrix composite" Acta. Metall. Mater. 38, 185-194.

Voigt, T., Ye, G., Sun, Z., Shah, S. P. \& van Breugel, K. (2005) "Early age microstructure of Portland cement mortar investigated by ultrasonic shear waves and numerical simulation" Cem. Concr. Res. 35, 858-866.
Zhonghua, L., Schmauder, S., Wanner, A. \& Dong, M. (1995) "Expression to characterize the flow behavior of particle-reinforced composites based on axi-symmentric unit cell modules" Scr. Metall. Mater. 1289-1294.

Zimmerman, R. W. (1984) "Elastic moduli of a solid with spherical pores: New self-consistent method" Int. J. Rock Mech. and Mining Sci. Geomech. Abst. 21 (6), 339-343.

(Date of Submission : 2010.9.9) 